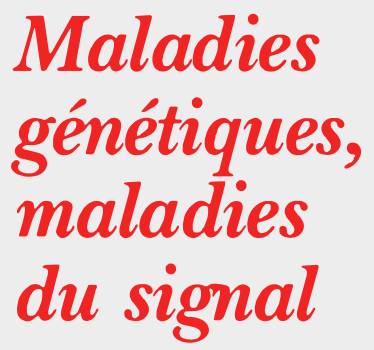

Les maladies génétiques étaient jadis attribuées selon le modèle classique de la génétique microbienne, soit à des mutations des gènes de structure, soit à des mutations des gènes de régulation. Dans la première catégorie, on peut ranger les défauts génétiques portant sur les protéines de structure, les enzymes du métabolisme, ou des transporteurs comme l'hémoglobine. La seconde est, en réalité, beaucoup plus vaste, si on entend par là toutes les altérations génétiques entraînant des anomalies de la régulation de l'expression génique, avec leurs conséquences sur le développement et la différenciation cellulaire. Outre les atteintes génétiques de la machinerie transcriptionnelle, on peut classer dans cette catégorie de très nombreuses anomalies de la transmission du signal, qu'il s'agisse d'une activation anormale ou d'un déficit. Ainsi, les mutations du récepteur RET sont-elles associées à une néoplasie endocrinienne multiple de type 2, ou à une maladie de Hirschprung, et leur pénétrance peut être modulée par les mutations du ligand de RET, le facteur GNDF. D'autres exemples de mutation de récepteurs à activité de tyrosine kinase avec craniosynostoses, d'un récepteur hormonal avec anomalie du développement sexuel et d'une phosphatase de phosphoinositides associée à un retard mental et à des malformations sont illustrés dans ce numéro de $\mathrm{m} / \mathrm{s}$. Chez certains malades, la sécrétion inappropriée du facteur BMP4, de mécanisme inconnu, conduit à une maladie ossifiante des parties molles.

\title{
RET et GDNF: un récepteur orphelin trouve une famille nourricière
}

Le sort des modèles en science est d'être pris en défaut. Sept articles publiés récemment dans Nature et Cell [1-7] le rappellent de manière opportune au lecteur et l'invitent à renoncer au bien-être procuré par des connaissances tenues pour acquises. A la suite de ces travaux, il est établi qu'un membre distant de la famille TGF- $\beta$ appelé GDNF (glial cell line-derived neurotrophic factor) est un ligand du récepteur tyrosine kinase RET. Il nous faut donc, d'une part, reconsidérer l'attribution univoque de récepteurs sérine/thréonine kinase aux membres de la famille TGF- $\beta$. D'autre part, il nous faut concevoir l'interaction entre un ligand et un récepteur doté d'activité tyrosine kinase (RTK), en l'occurrence le GDNF et RET, comme plus subtile que nous ne la soupçonnions. Celle-ci peut en effet requérir la fixation préalable du ligand sur un co-récepteur, condition nécessaire à la formation d'un complexe compétent pour la transduction de signaux. Finalement, ces résultats illustrent l'apport fécond de la mutagenèse ciblée in vivo (knock-out) à différents champs de la recherche en biologie dont l'embryologie et la génétique des maladies humaines.

\section{RET : un récepteur tyrosine kinase} atypique

Le produit du proto-oncogène $R E T$ est une glycoprotéine transmembranaire pourvue d'un domaine tyrosine kinase intracytoplasmique (pour revue voir $[8,9])$. Au premier abord, rien ne distingue RET des autres membres de la famille RTK. Comme pour nombre de ses pairs la diversité est de mise, et le gène RET humain code pour plusieurs variants engendrés par épissage alternatif, en particulier trois isoformes comprenant 1114,1106 et 1072 acides aminés qui divergent dans leur région carboxy-terminale [10]. D'autre part, propriété commune à de nombreux RTK, la protéine RET est dotée d'un motif juxtamembranaire riche en cystéines. Cependant, RET se singularise par la présence d'un domaine de 110 acides aminés ayant une homologie significative avec les cadhérines, des molécules d'adhérence dont le domaine extracellulaire comprend une répétition en tandem de 5 à plus de 40 motifs capables de fixer le calcium. L'existence d'un tel motif dans le domaine extracellulaire de RET suggère, par analogie, que cette protéine puisse se comporter comme une cadhérine, quoique ses propriétés adhésives dépendantes du calcium restent encore à démontrer.

\section{RET, embryopathie et cancers}

Les lecteurs de médecine/sciences ont été informés des progrès récents de la recherche sur les causes génétiques de deux maladies humaines, à savoir les néoplasies endocriniennes multiples de type 2 (NEM 2) et la maladie de Hirschsprung [11]. Dans le cadre de cette mini-synthèse consacrée à RET et à son ligand, nous ne pouvons pas rentrer dans les détails de la sémiologie et de la génétique de ces deux affections. Aussi, nous souhaiterions nous concentrer sur quelques résultats en rapport avec le sujet (nous invitons le lecteur à se reporter à la revue précitée [11], ainsi qu'à d'autres synthèses plus récentes $[8,9])$. 
Les patients atteints de NEM 2 développent diverses tumeurs endocrines dont le cancer médullaire de la thyroïde (CMT) et le phéochromocytome. Les NEM 2 sont des multiendocrinopathies héréditaires à mode de transmission autosomique dominant et à forte pénétrance [8, 9]. La maladie de Hirschsprung est une malformation digestive congénitale caractérisée par l'absence des ganglions entériques (sous-muqueux et myentérique) dans une portion plus ou moins étendue de l'intestin. Les symptômes de cette aganglionose sont une occlusion intestinale néonatale grave ou une constipation chez l'adulte. L'étude des formes familiales de maladie de Hirschprung a permis de mettre en évidence plusieurs gènes responsables, dont un gène autosomique dominant de pénétrance incomplète $[8,9,11]$.

Des mutations germinales du gène RET situé sur le chromosome 10 (10q11.2) sont la cause des NEM 2 et d'une majorité des formes familiales de Hirschsprung avec un mode de transmission dominant. Dans les NEM 2, ces mutations sont des mutations ponctuelles dont les conséquences sont, soit la substitution d'une cystéine du domaine riche en cystéines par un autre acide aminé, soit le remplacement d'une méthionine particulière du domaine tyrosine kinase par une thréonine $[8,9$, 11]. Quant à la maladie de Hirschsprung, les mutations en cause peuvent être la délétion d'un allèle, des mutations qui décalent le cadre de lecture, des mutations non-sens ou des mutations faux-sens réparties sur l'ensemble de la région codante [8, $9,11]$. Les altérations génétiques associées à ces deux maladies sont donc de nature différente et ont des conséquences opposées sur la fonction de RET. En effet, les mutations des NEM 2 conduisent à une activation constitutive de l'activité tyrosine kinase et transforment le gène $R E T$ en oncogène (gain de fonction), alors que les mutations Hirschsprung aboutissent dans la majorité des cas à une haplo-insuffisance due à l'absence d'expression d'un allèle ou à l'expression d'une protéine non fonctionnelle (perte de fonction $[8,9])$

\section{RET, organogenèse rénale et développement du système nerveux périphérique}

Au cours du développement, le gène $R E T$ est exprimé de manière dynamique dans plusieurs territoires embryonnaires, dont le système nerveux et le rein en voie de formation $[12,13]$. Concernant le système nerveux, l'expression de RET est en particulier détectable dans les cellules de la crête neurale progénitrices du système nerveux entérique ( $\mathrm{SNE}$ ), dans les ganglions crâniens, les ébauches des ganglions sympathiques et rachidiens et dans la partie ventrale du tube neural d'où proviennent les motoneurones $[12,13]$. Lors de l'édification du métanéphros, RET est exprimé exclusivement à la surface des cellules épithéliales situées à l'extrémité du bourgeon urétéral, bourgeon qui s'invagine dans le blastème métanéphrogène puis se divise par bifurcations successives pour former les éléments excréteurs du rein définitif $[12,13]$. Les expériences de mutagenèse ciblée in vivo chez la souris ont clairement confirmé les données d'expression qui suggéraient un rôle de RET durant l'embryogenèse [14]. En effet, les souris dont les deux allèles Ret ont été invalidés meurent dans la journée qui suit leur naissance. Chez ces animaux nullizygotes $\left(\right.$ Ret $\left.^{-1}\right)$ le SNE est absent dans l'ensemble de l'intestin, de plus le ganglion cervical supérieur (GCS) est manquant et ces souris ont, soit des reins très rudimentaires, soit une agénésie rénale (Tableau I, $[14,15])$. Les souris $\operatorname{Ret}^{-/-}$constituent donc un modèle animal de maladie de Hirschsprung, et ce modèle valide à rebours le rôle étiologique des mutations de RET dans cette maladie.

\section{Quel ligand pour RET?}

Ces résultats remarquables et des travaux ultérieurs ont conduit à la formulation de plusieurs hypothèses relatives à la nature du ligand de $\operatorname{RET}[15,16]$. Tout d'abord, il s'avère qu'une sous-population des cellules de la crête neurale vagale contribue de manière majeure à la formation du SNE intestinal et du GCS. Ces cellules aux potentialités entérique et sympathique dépendraient de RET et de sa capacité de recevoir un signal approprié pour se développer normalement [15]. D'autre part, un ensemble d'arguments expérimentaux suggère que l'absence du GCS et probablement aussi du SNE chez les souris $R e t^{-/-}$n'est pas due à un défaut de migration des cellules appartenant au lignage sympathoentérique de la crête neurale, mais peut être plutôt attribuée à un événement postérieur survenant au cours de la gangliogenèse. D'où la première supposition que le ligand de RET puisse être un facteur trophique [15]. L'analyse des causes potentielles de la dysgénésie rénale des souris $\mathrm{Ret}^{-1-}$ inspire de nouvelles réflexions. En effet, le rein se forme à partir du blastème métanéphrogène et du bourgeon urétéral. Tandis que, sous l'influence inductive des cellules mésenchymateuses du blastème, le bourgeon urétéral se divise en bifurcations dichotomiques jusqu'à former les grands et petits calices et les tubules collecteurs, sous l'influence inductive du bourgeon urétéral les cellules du mésenchyme vont se condenser en amas qui constitueront des vésicules rénales puis finalement les néphrons. Des expériences de co-culture de bourgeon urétéral et de mésenchyme métanéphrique démontrent sans ambiguïté que RET est requis dans les cellules du bourgeon urétéral pour la transmission du signal inducteur provenant du blastème [16] D'où la deuxième supposition que le ligand de RET pourrait être un facteur doté de propriétés inductrices $[14,16]$

\section{Le GDNF : pléiotropie d'une neurotrophine}

L'observation que du milieu conditionné de cellules gliales exerçait une activité trophique sur les neurones dopaminergiques d'origine mésencéphalique a conduit Frank Collins et ses collaborateurs (Synergen, Inc, Boulder, CO, USA) en 1993 à purifier le facteur responsable de cet effet et à cloner son gène [17]. Ce facteur appelé GDNF (glial cell linederived neurotrophic factor) présente une homologie d'environ $20 \%$ avec 
Tableau I

COMPARAISON DU PHÉNOTYPE DES SOURIS PORTEUSES

DE MUTATIONS INACTIVATRICES DANS LES GĖNES Ret ET Gdnf

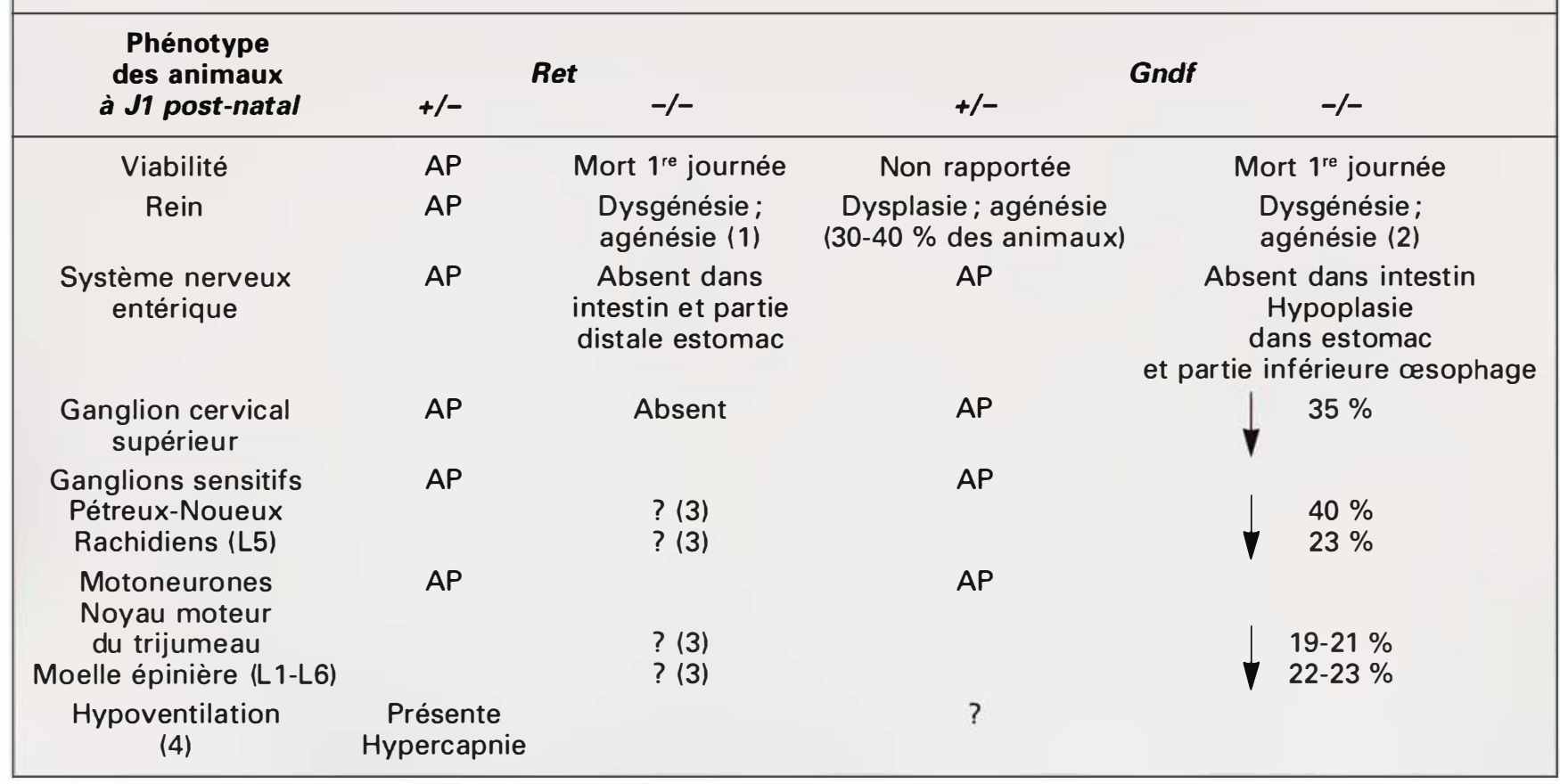

Résultats prenant en compte les données publiées dans les articles 3, 4, 5. +/- et -l- symbolisent l'hétérozygotie et l'homozygotie des animaux dont le gène a été invalidé. AP: absence de phénotype. $\downarrow$ : Diminution du nombre de neurones exprimée en \%.

(1) Agénésie rénale souvent accompagnée d'absence de l'uretère. Chez les animaux Ret'- deux reins très rudimentaires sont présents dans $11 \%$ des cas; agénésie rénale unilatérale et rein rudimentaire: $31 \%$ des cas; agénésie rénale bilatérale: $58 \%$ des cas. Vingt-cinq pour cent des femelles Ret' présentent aussi des malformations de l'utérus.

(2) Agénésie rénale associée à une absence de l'uretère.

(3) Analyse en cours dans le laboratoire de V. Pachnis (Londres, Grande-Bretagne).

(4) Résultats préliminaires qui n'ont fait l'objet que de communications dans des congrès.

les protéines de la superfamille des cytokines TGF- $\beta[17,18]$. De plus, les sept cystéines impliquées dans la structuration du motif cystine knot des molécules TGF- $\beta$ [19] sont présentes dans le GDNF. Comme les autres membres de cette famille, le GDNF est synthétisé sous la forme d'un précurseur qui, après clivage, engendre une protéine mûre de 134 acides aminés. Le produit sécrété et biologiquement actif est un dimère composé de deux molécules de GDNF reliées entre elles par un (ou des) pont disulfure [17]. Outre sa capacité d'assurer la survie des neurones dopaminergiques, le GDNF exerce une activité trophique sur les motoneurones, les neurones noradrénergiques du locus coruleus et différentes classes de neurones du système nerveux périphérique [20- le GDNF est un facteur neurotrophique aux applications cliniques potentielles dans le cadre d'affections neurodégénératives, en particulier la maladie de Parkinson [28] et la sclérose latérale amyotrophique (m/s $n^{\circ} 4$, vol. 12, p. 544) [29].

Durant l'embryogenèse, le GDNF est exprimé dans les cordons néphrogènes, l'intestin primitif et les poches entobranchiales [3-5, 30]. A des stades plus tardifs le transcrit est détectable dans le blastème métanéphrogène, le mésenchyme de l'ensemble du tractus digestif, les bourgeons des membres et dans les régions ventrales du mésencéphale et du rhombencéphale [3-5, 30]. Le GDNF pourrait-il être plus qu'un facteur neurotrophique comme le suggère son profil d'expression? Le bien-fondé de cette supposition a été confirmé par trois équipes qui, indé- pendamment, ont introduit par mutagenèse ciblée une mutation nulle dans le gène $G d n f$ [3-5]. De manière surprenante, $30 \%$ à $40 \%$ des souris hétérozygotes $\left(G d n f^{+/-}\right)$ont de graves malformations rénales (Tableau I). Les animaux homozygotes $\left(G d n f^{-1-}\right)$ meurent au cours de la première journée post-natale. Ces souris $G d n f^{-/-}$présentent dans la majorité des cas une agénésie rénale bilatérale consécutive à l'incapacité du bourgeon urétéral de se former correctement [4]. D'autre part, les ganglions intramuraux de l'intestin sont absents, et le SNE de l'estomac est, soit manquant, soit hypoplasique [3-5]. Le GCS est présent, mais le nombre de neurones est réduit d'environ un tiers $[3,5]$. Le nombre de motoneurones du noyau moteur du trijumeau et de la moelle lombaire et celui de différents types de 
ganglions sensitifs est aussi significativement diminué (Tableau I) $[3,5]$. En revanche, les neurones dopaminergiques du locus niger et les neurones noradrénergiques du locus cœruleus ne semblent pas affectés $[3,5]$. Au vu de ces données nous sommes fondés à conclure que le GDNF est un des facteurs inducteurs sécrété par le blastème métanéphrogène dont la présence est indispensable à la croissance et à l'arborisation du bourgeon urétéral. Par ailleurs, il est vraisemblable que le GDNF exerce au cours du développement une activité trophique sur différentes populations de neurones, en particulier sur les cellules de la crête neurale vagale lors de leur colonisation de l'intestin embryonnaire.

\section{Quel récepteur pour le GDNF?}

Les récepteurs des membres de la famille TGF- $\beta$ constituent une famille de récepteurs transmembranaires avec un domaine sérine/thréonine kinase intracytoplasmique [31]. Il était donc a priori logique d'inférer que le récepteur GDNF pût appartenir à cette famille. Mais les a priori sont fondés sur des modèles faillibles, et la réponse s'avère différente. Grâce aux criblages de banques d'expression avec du GDNF marqué à l'iode 125, deux groupes [6, 7] ont cloné un ADNc codant pour une protéine liant le GDNF avec une forte affinité ( $\mathrm{Kd}$ compris entre 2,0 $010^{-12}$ $\mathrm{M}$ et $\left.6,0 \times 10^{-11} \mathrm{M}\right)$. Cette protéine dénommée GDNFR $\alpha$ comprend 465 acides aminés chez l'homme [7] et est liée à la membrane plasmique par une ancre GPI (glycosyl phosphatidyl inositol (voir figure 1). Le gène GDNFR- $\alpha$ est exprimé au cours de l'ontogenèse de nombreux organes dont le rein et le tube digestif [6]. L'absence de domaine intracytoplasmique capable de transmettre un signal dans la cellule indiquait que le GDNFR- $\alpha$ était un des partenaires d'un complexe multiprotéique encore incomplètement caractérisé. L'expression en temps et lieux du GDNF dans les tissus en voie de formation et censés exprimer le ligand de RET, mais surtout l'étonnante similitude entre le phénotype des souris $G d n f^{\prime-}$ et Ret $^{-/-}$(Tableau I) suggéraient que

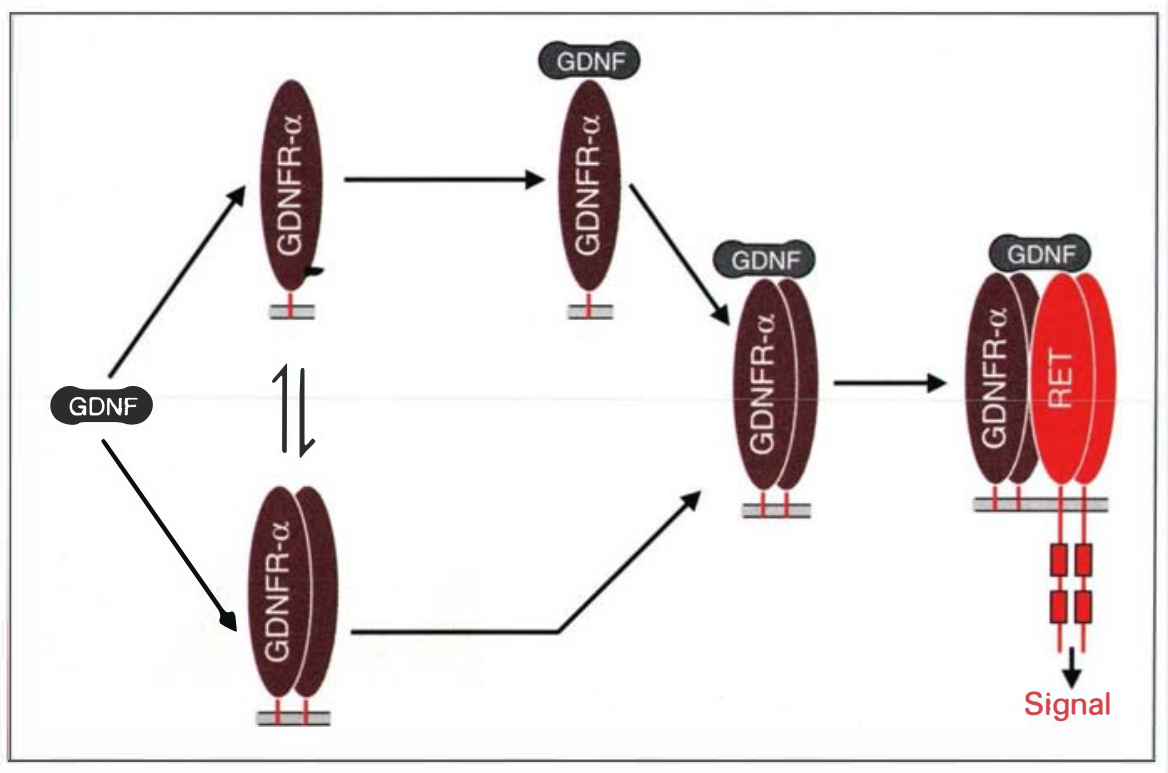

Figure 1. Formation du complexe comprenant GDNF, GDNFR- $\alpha$ et RET. (D’après [7].) Le premier événement déclenchant la formation du complexe capable de transmettre le signal serait la fixation du GDNF dimérique sur le co-récepteur GDNFR- $\alpha$. Dans la mesure où il n'a pas été montré de manière formelle que le GDNF puisse induire la dimérisation de GDNFR- $\alpha$, il n'est pas exclu que les deux espèces de GDNFR- $\alpha$ (monomérique et dimérique) existent en équilibre à la surface de la cellule. Cette alternative a été figurée sur le schéma. Le deuxième événement serait l'induction de l'homodimérisation de RET consécutive à l'interaction de deux monomères RET avec le complexe GDNF/GDFNR- $\alpha$. La dimérisation de RET conditionnerait l'activation de la tyrosine kinase et la transmission du signal qui en découle.

RET pût être un autre élément du puzzle. Cette hypothèse a été testée et validée par quatre des groupes dont nous décrivons ici les travaux $[1,2,6,7]$. En effet, RET lie le GDNF et cette fixation active la tyrosine kinase. En outre, le GDNF stimule la prolifération de fibroblastes synthétisant RET de manière exogène [1], induit la formation dépendante de RET de mésoderme dans une structure à devenir épithélial de la blastula de Xénope [2], et promeut la croissance axonale de neuroblastes présents dans des explants uro-génitaux embryonnaires murins mais non dans le même type d'explant prélevé chez des souris Ret $^{-1-}$ [2].

Si le GDNF se lie à RET, quel est le rôle du GDNFR- $\alpha$ ? En fait, l'élimination du GDNFR- $\alpha$ de la surface des cellules par action d'une phospholipase, prévient l'activation de RET par le GDNF [6, 7]. D'autre part, l'ajout de GDNFR- $\alpha$ soluble au GDNF potentialise l'autophosphorylation de
RET. Finalement, des expériences d'immunoprécipitation réalisées après pontage covalent des protéines de surface révèlent l'existence de différents complexes composés de GDNF, GDNFR- $\alpha$ et RET [6, 7]. Il apparaît donc probable (le conditionnel est de mise car il existe certaines contradictions entre les articles, voir [1] et [6, 7]) que la liaison et l'activation de RET par le GDNF nécessitent la présence du GDNFR- $\alpha$. Dans des conditions physiologiques la dimérisation des RTK, dont RET, est requise pour l'activation de la tyrosine kinase. Par conséquent, la stœechiométrie du complexe capable de transmettre le signal pourrait être: un dimère de GDNF, deux molécules de GDNFR- $\alpha$ et deux molécules de RET (figure 1). Si tel était le cas, la nature de ce complexe serait proche de ceux déjà connus et impliqués dans la réception de l'interleukine 6 et du CNTF (ciliary neurotrophic factor) [32]. 


\section{Épilogue}

La pertinence expérimentale de ces résultats est incontestable, et la double propriété trophique et inductrice postulée pour le ligand de RET est imputable au GDNF. Il reste cependant des lacunes, certaines incohérences et des données préliminaires qui font penser que le sujet n'est pas clos et recèle des surprises dont, à n'en pas douter, de futures synthèses dans médecine/sciences. feront l'objet.

En premier lieu, le rôle de RET dans les motoneurones et les neurones spinaux embryonnaires ainsi que son expression et sa fonction dans l'encéphale adulte sont des questions encore mal étudiées. Différentes approches, dont l'invalidation ciblée à certaines structures nerveuses du gène $R E T$ grâce au système de recombinaison spécifique Cre-loxP $[33,34]$, sont envisageables pour répondre à quelques-unes de ces interrogations. D'autre part, il est plausible que RET, comme les RTK de la famille Trk [35], ait plus d'un ligand. Une telle éventualité permettrait d'expliquer pourquoi les animaux $G d n f^{-1-}$ ont une réduction partielle du GCS comparés aux souris Ret ${ }^{-1-}$ (TableauI). Cette hypothèse est étayée par la caractérisation récente de deux nouveaux facteurs neurotrophiques, nommées persefine et neurturine, et dont la séquence en acides aminés est très proche de celle du GDNF [36].

En deuxième lieu, il reste à étudier si le GDNF assure uniquement la survie des neurones entériques, ou s'il est impliqué plus largement dans la prolifération et la différenciation de ces cellules. Par ailleurs, l'absence de GDNF ne semble pas altérer le développement des neurones dopaminergiques de la substantia nigra. Si durant l'embryogenèse le GDNF n'est pas un facteur limitant pour cette classe de neurones, d'autres molécules aux propriétés analogues peuvent probablement exercer un tel effet trophique. Quelles sont-elles? Notons que trois membres de la famille TGF- $\beta$ sont capables de prévenir la dégénérescence des neurones dopaminergiques [37, 38]. Finalement, des mutations du gène $R E T$ ont familiales de maladie de Hirschsprung (en prenant en compte les formes autosomique dominante et autosomique récessive) et dans 15 à $20 \%$ des cas sporadiques. Les mutations dans d'autres gènes comme ceux codant pour l'endothéline 3 et le récepteur bêta des endothélines sont probablement responsables d'un petit nombre de cas de Hirschsprung [39], ce qui suggère l'existence d'un ou plusieurs autres locus de prédisposition. Les gènes GDNF et GDNFR- $\alpha$ constituent donc d'excellents candidats pour la maladie de Hirschsprung, ainsi que pour les formes familiales d'agénésie rénale

Remerciements

Nous remercions P. Durbec pour nous avoir fait profiter de ses connaissances lors de la rédaction de cette revue.

\section{Sophie Chappuis \\ Olivier Geneste \\ Andrea Pasini \\ Gilbert Lenoir \\ Marc Billaud}

Laboratoire de Génétique, Cnrs UMR 5641, Domaine Rockfeller. 8, avenue Rockfeller, 69373 Lyon Cedex 08, France.

\section{TIRÉS À PART}

M. Billaud.

\section{RÉFÉRENCES}

1. Trupp M, Arenas E, Fainzilber M, Nilsson AS, Sieber BA, Grigoriou M, Kilkenny $C_{\text {, }}$ Salazar-Grueso E, Pachnis V, Arumäe U, Sariola H, Saarma M, Ibañez CF. Functional receptor for GDNF encoded by the c-ret proto-oncogene. Nature 1996; 381: 785-9.

2. Durbec P, Marcos-Gutierrez CV, Kilkenny C, Grigoriou M, Wartiowaara $K$, Suvanto $P$ Smith D, Ponder B, Costantini F, Saarma M, Sariola $H$, Pachnis V. GDNF signalling through the RET receptor tyrosine kinase Nature 1996; 381 : 789-93.

3. Sanchez MP, Silos-Santiago I, Frisén J, He B, Lira SA, Barbacid M. Renal agenesis and the absence of enteric neurons in mice lacking GDNF. Nature 1996; 382: 70-3.

4. Pichel JG, Shen L, Sheng HZ, Granholm AC, Drago J, Grinberg A, Lee EJ, Huang SP Saarma M, Hoffer BJ, Sariola H, Westphal $H$. Defects in enteric innervation and kidney development in mice lacking GDNF. Nature $1996 ; 382$ : 73-6.

5. Moore MW, Klein RD, Fariñas I, Sauer H, Armanini M, Phillips H, Reichardt LF, Ryan AM, Carver-Moore K, Rosenthal A. Renal and neuronal abnormalities in mice lacking GDNF. Nature 1996; 382: 76-9.

6. Treanor JIS, Goodman L, de Sauvage F, Stone DM, Poulsen KT, Beck CD, Gray C, Armanini MP, Pollock RA, Hefti F, Phillips HS, Goddard A, Moore MW, Buj-Bello A, Davies AM, Asai N, Takahashi M, Vandlen R, Henderson CE, Rosenthal A. Characterization of a multicomponent receptor for GDNF. Nature 1996; 382: 80-3.

7. Jing S, Wen D, Yu Y, Holst PL, Luo Y, Fang M, Tamir R, Antonio L, Hu Z, Cupples R, Louis JC, Hu S, Altrock BW, Fox GM. GDNFinduced activation of the RET protein tyrosine kinase is mediated by GDNFR- $\alpha$, a novel receptor for GDNF. Cell 1996; 85: 1113-24.

8. Goodfellow PJ, Wells Jr SA. RET gene and its implication for cancer. J Natl Cancer Instit 1995; 87: 1515-23.

9. Pasini B, Ceccherini I, Romeo G. RET mutations in human disease. Trends Genet $1996 ; 12$ : 138-44.

10. Myers SM, Eng C, Ponder BAJ, Mulligan LM. Characterization of RET proto-oncogene 3' splicing variants and polvadenylation sites: a novel C-terminus for RET. Oncogene 1995; 11 : 2039-45.

11. Lyonnet S, Edery P, Attié T, NihoulFékété C, Munnich A et le Consortium Français de la Maladie de Hirschsprung. Des mutations du proto-oncogène RET dans la maladie de Hirschprung: un gène à tout faire. médecine/sciences 1994; 4: 450-3.

12. Pachnis V, Mankoo B, Costantini F. Expression of the c-ret proto-oncogene during embryogenesis. Development 1993; 119: 1005-17.

13. Tsuzuki $T$, Takahashi $M$, Asai N, Iwashita T, Matsuyama M, Asai J. Spatial and temporal expression of the ret proto-oncogene product in embryonic, infant and adult rat tissues. Oncogene 1995; $10: 191-8$.

14. Schuchardt A, D'Agati V, Larsson-Blomberg L, Costantini F, Pachnis V. Defects in the kidney and enteric nervous system of mice lacking the tyrosine kinase receptor Ret. Nature 1994; 367: 380-3.

15. Durbec PL, Larsson-Blomberg LB, Schuchardt A, Costantini F, Pachnis V. Common origin and developmental dependence on c-ret of subsets of enteric and sympathetic neuroblasts. Development 1996; 122: 349-58. 16. Schuchardt A, D'Agati V, Pachnis V, Costantini F. Renal agenesis and hypodysplasia in ret-k- mutant mice result from defects in ureteric bud development. Development 1996; 122: 1919-29.

17. Lin LFH, Doherty DH, Lile JD, Bektesh S, Collins F. GDNF: a glial cell line-derived neurotrophic factor for midbrain dopaminergic neurons. Science 1993; 260 : 1130-2.

18. Kingsley DM. The TGF- $\beta$ superfamily: new members, new receptors, and new genetic tests of function in different organisms. Genes Dev 1994; 8: 133-46.

19. McDonald NQ Hendrickson WA. A structural superfamily of growth factors containing a cystine knot motif. Cell 1993; 73: 421-4.

20. Henderson CE, Phillips HS, Pollock RA, Davies AM, Lemeulle C, Armanini M, Simpson LC, Moffet B, Vandlen RA, Koliastos VE, 
Rosenthal A. GDNF : a potent survival factor for motoneurons present in peripheral nerve and muscle. Science 1994; 266: 1062-4. 21. Tomac A, Lindqvist E, Lin LFH, Ögren SO, Young D, Hoffer BJ, Olson L. Protection and repair of the nigrostriatal dopaminergic system by GDNF in vivo. Nature 1995 ; 373: 335-9.

22. Beck KD, Valverde I, Alexi T, Poulsen K, Moffat B, Vandlen RA, Rosenthal A, Hefti F. Mesencephalic dopaminergic neurons protected by GDNF from axotomy-induced degeneration in the adult brain. Nature 1995; 373: 339-41.

23. Yan Q, Matheson C, Lopez OT. In vivo neurotrophic effects of GDNF on neonatal and adult facial motor neurons. Nature $1995 ; 373$ : 341-4.

24. Oppenheim RW, Houenou LJ, Johnson JE, Lin LFH, Li L, Lo AC, Newsome AL, Prevette DM, Wang S. Developing motor neurons rescued from programmed and axotomy-induced cell death by GDNF. Nature $1995 ; 373$ : 344-6.

25. Trupp $M$, Rydén $M$, Jörnvall $\mathrm{H}$, Funakoshi H, Timmusk T, Arenas E, Ibañez CF. Peripheral expression and biological activities of GDNF, a new neurotrophic factor for avian and mammalian peripheral neurons. $J$ Cell Biol 1995; 130: 137-48.
26. Arenas E, Trupp M, Åkerud P, Ibañez CF. GDNF prevents degeneration and pro motes the phenotype of brain noradrenergic neurons in vivo. Neuron 1995; 15: 1465-73.

27. Buj-Bello A, Buchman VL, Horton A Rosenthal A, Davies AM. GDNF is an agespecific survival factor for sensory and autonomic neurons. Neuron 1995; 15: 821-8.

28. Gash DM, Zhang Z, Ovadia A, Cass WA, Yi A, Simmerman L, Russell D, Martin D Lapchak PA, Collins F, Hoffer BJ, Gerhard GA. Functional recovery in parkinsonian monkeys treated with GDNF. Nature 1996 380: 252-5.

29. Heyd D, Aebischer P. Les facteurs neurotrophiques et leurs applications thérapeutiques potentielles. médecine/sciences 1996 ; 12 : 299-302.

30. Suvanto $P$, Hiltunen JO, Arumãe U, Moshnyakov M, Sariola H, Sainio K, Saarma M. Localization of glial cell line-derived neurotrophic factor (GDNF) mRNA in embryonic rat by in situ hybridization. Eur J Neurosci 1996; 8: 816-22.

31. ten Dijke P, Miyazono K, Heldin CH. Signalling via hetero-oligomeric complexes of type $\mathbf{I}$ and type II serine/threonine kinase receptors. Curr Op Cell Biol 1996; 8 : $139-45$.

32. Dusanter-Fourt I, Mayeux P, Gisselbrecht $S$. Transduction du signal par les récepteurs de cytokines. médecine/sciences 1994; 10: 825-35.

33. Babinet C. Cre-LoxP et cellules souches embryonnaires. médecine/sciences $1995 ; 8$ : 1154-7.

34. Viville S. Recombinaison homologue: nouveaux vecteurs, nouvelles perspectives. médecine/sciences $1995 ; 11$ : 735-46.

35. Lamballe F. Les récepteurs tyrosine kinases Trk: récepteurs de forte affinite des neurotrophines. médecine/sciences $1995 ; 8$ : 1071-80.

36. Kotzbauer PT, Lampe PA, Johnson EM, Milbrandt J. Signalling in neuronal development, differentiation and degeneration, Keystone Symposia on Molecular and Cellular Biology. Taos, NM, 27 mars-2 avril 1996, p 136. 37. Poulsen KT, Armanini MP, Klein RD, Hynes MA, Phillips HS, Rosenthal A. TGF beta 2 and TGF beta 3 are potent survival factors for midbrain dopaminergic neurons. Neuron 1994; 13: 1245-52.

38. Krieglstein K, Suter-Crazzolara C, Fisher WH, Unsicker K TGF-beta superfamily members promote survival of midbrain dopaminergic neurons and protect them against $M^{2}{ }^{+}$toxicity. $E M B O J 1995 ; 14: 736-42$.

39. Attié T, Amiel J, Edery P, Lyonnet $S$. Endothélines et leurs récepteurs : de nouveaux acteurs du développement. médecine/sciences $1995 ; 11$ : 1172-5. 